\title{
Verkürzte Liegezeiten und Probleme hausärztlicher Anschlussversorgung:
}

\section{Eine qualitative Fallstudie zum Sektorenübergang}

\section{SUSANNE GRUNDKE, \\ GITTA KUDELA, ANDREAS KLEMENT}

Prof. Dr. phil. Susanne Grundke ist gelernte Altenpflegerin und Professorin für angewandte Pflegewissenschaft mit Schwerpunkt Pflegebildung im Department Gesundheit und Pflege der Hochschule für Technik und Wirtschaft des Saarlandes;

Dr. med. Gitta Kudela ist niedergelassene Fachärztin für Allgemeinmedizin, langjähriges Mitglied der Fachkommission Allgemeinmedizin der Ärztekammer Sachsen-Anhalt und aktiv in der Studentenausbildung als Akademische Lehrpraxis für Allgemeinmedizin der Universität Magdeburg;

Prof. Dr. med. Andreas Klement ist niedergelassener Facharzt für Allgemeinmedizin und leitet die Sektion Allgemeinmedizin der Medizinischen Fakultät der Universität Halle-Wittenberg.

\author{
Qualitative Fallanalysen auf Grundlage von \\ Interviews mit Hausärzten, können aktuell (fort-) \\ bestehende Schnittstellenprobleme beim stationär- \\ ambulanten Sektorenübergang abbilden. Die \\ Ergebnisse unserer Befragung zeigen, dass nicht \\ eine kürzere stationäre Verweildauer, sondern \\ eine zu späte oder fehlende Übermittlung \\ behandlungswichtiger Informationen als zentrales \\ Problem des Entlassungsmanagements beschrieben \\ wird. Unzureichende entlassungsvorbereitende \\ Absprachen mit Hausarzt und Angehörigen gefährden
die Kontinuität der Versorgung vulnerabler Patienten.
}

\section{Hintergrund}

Der Sachverständigenrat der Bundesregierung für die Beurteilung der Entwicklung im Gesundheitswesen (SVR) hat in seinem Jahresgutachten 2012 den Wettbewerb an der Schnittstelle zwischen ambulanter und stationärer Gesundheitsversorgung analysiert. Die Sicherstellung von Versorgungskontinuität wird darin als Kernaufgabe des Schnittstellenmanagements beim stationär-ambulanten Übergang angesehen [1]. Schon in seinen Gutachten 2003 \& 2007 hatte der SVR festgestellt, dass besonders Patienten im höheren Lebensalter mit ausgeprägter Multimorbidität einen zunehmend komplexen Versorgungsbedarf nach Entlassung aufweisen und durch Versorgungsdiskontinuitäten besonders gefährdet sind [2]. Im Versorgungsstrukturgesetz vom 01.01.2012 wurde entsprechend das Entlassungsmanagement als obligatorischer Teil des Anspruchs auf Krankenhausbehandlung konkretisiert (\$ 39 GKV-VStG).
Seit Einführung des DRG-Entgeldsystems in Deutschland (G-DRG) haben sich die stationären Verweildauern erheblich verkürzt und stationäre Arbeitsabläufe verdichtet. Strittig ist weiterhin, ob dadurch Teile der Behandlung und des Genesungsprozesses in den nachstationären Bereich verlagert werden [3]. Die gesetzlich geforderte offizielle DRG-Begleitforschung konnte anhand nachstationärer Arztkontaktzahlen und Hauskrankenpflegekosten bisher über demographische Effekte hinaus keine signifikante Leistungsverlagerung in den ambulanten Sektor feststellen, verweist aber auf den Mangel geeigneter Indikatoren zur Abbildung von zunehmender nachstationärer Behandlungskomplexität [4]. Gesichert ist, dass aus demographischen Gründen erhöhter nachstationärer Versorgungsbedarf durch verkürzte Liegezeiten weiter zunimmt. Eine strukturierte Entlassungsplanung ist daher notwendig, um bei vulnerablen Patienten Versorgungsbrüche beim Wechsel der Versorgungsebenen zu vermeiden [1]. Koordination und Kom- 
munikation zwischen den Leistungserbringern nehmen dabei eine Schlüsselfunktion ein [5]. Die Effektivität einer strukturierten und individualisierten Entlassungsplanung im Hinblick auf verkürzte Verweildauern und ungeplante Wiederaufnahmen bei älteren Patienten ist dokumentiert; nicht belegt ist ob dadurch auch Mortalitätsraten und Kosten gesenkt werden können [6].

Gegenüber dem SVR gaben 2011 in einer aktuellen bundesweiten Befragung $78 \%$ der Krankenhäuser an, ein strukturiertes Entlassungsmanagement durchzuführen, in 45\% der Häuser sei ein „Expertenstandard Entlassungsmanagement in der Pflege“ bereits eingeführt worden [1]. In einer Längsschnittanalyse berichtete jedoch eine Mehrheit der Pflegekräfte 2006 gegenüber 2003 eine Verschlechterung der Situation im Entlassungsmanagement, was von zwei Dritteln der zuweisenden Hausärzten mit wahrgenommener „blutiger Entlassung“ und „Drehtüreffekten“ in Verbindung gebracht wird [7, 8]. Pflegekräfte und Ärzte in Krankenhäusern geben seit der DRG-Einführung eine zunehmende Belastung durch bürokratische und organisatorische Veränderungen an, die letztlich zu weniger verfügbarer Zeit für den Patientenkontakt und Kommunikation führten [8]. Patienten hingegen erwarten ausdrücklich beim Schnittstellenübergang, dass eine nahtlose ambulante Weiterbehandlung durch ärztlichen Bericht (z.B. Entlassungsbrief) gewährleistet wird und ihre individuellen Unterstützungsbedarfe/Informationsbedürfnisse zur Weiterbehandlung schon vor Entlassung berücksichtigt werden [9]. Ebenso wünschen Hausärzte von Kliniken eine Intensivierung gegenseitiger Information vor, während und am Ende (ungeplanter) stationärer Aufenthalte, insbesondere wenn kritische Ereignisse eintreten oder Langzeit-Therapien begonnen bzw. beendet werden [10].

Das Ziel unserer Studie ist es, bestehende Überleitungsprobleme aus hausärztlicher Sicht im Hinblick auf das Entlassungsmanagement bei verkürzten Liegezeiten abzubilden und Potenziale zur Verbesserung des stationär-ambulanten Überganges aufzuzeigen.

\section{Methode}

Aufgrund unzureichend verfügbarer quantitativer Daten bzw. Erhebungsin-
Tabelle 1: Beschreibung der Interview-Teilnehmer

\begin{tabular}{|c|c|}
\hline Teilnehmer & 16 \\
\hline $\begin{array}{l}\text { Geschlecht } \\
\text { weiblich } \\
\text { männlich }\end{array}$ & $\begin{array}{r}10 \\
6\end{array}$ \\
\hline $\begin{array}{ll}\text { Alter } & \\
\text { zwischen } & 30-45 \text { Jahren } \\
& 46-55 \text { Jahren } \\
& 56-65 \text { Jahren }\end{array}$ & $\begin{array}{l}4 \\
8 \\
4\end{array}$ \\
\hline $\begin{array}{l}\text { Gebietsweiterbildung } \\
\text { Facharzt für Allgemeinmedizin } \\
\text { Facharzt für Innere Medizin }\end{array}$ & $\begin{array}{r}14 \\
3\end{array}$ \\
\hline $\begin{array}{l}\text { Ort der Niederlassung } \\
\text { Städtische Region (>50 ooo Einwohner) } \\
\text { Ländliche Region (<50 ooo Einwohner) }\end{array}$ & $\begin{array}{r}10 \\
6\end{array}$ \\
\hline
\end{tabular}

strumente zu komplexen Überleitungsproblemen wurde eine explorative qualitative Studie durchgeführt [11]. In der Struktur eines Experteninterviews wurde mittels teilstandardisierten Interviewleitfadens um Fallerzählungen gebeten (u.a. „Bitte beschreiben Sie anhand eines Falls aus Ihrer Praxis wiederkehrend auftretende Schwierigkeiten in der Patientenüberleitung...“) und aufgezeichnet [12]. Es beteiligten sich von 25 schriftlich angefragten Moderatoren hausärztlicher Qualitätszirkel Sachsen-Anhalts bis Ende 2010 insgesamt 16 Ärzte mit 41 Fallerzählungen (Tabelle 1).

Die Interviews wurden im Originalwortlaut verschriftlicht (transkribiert). Die Auswertung erfolgte entsprechend der Erkenntnislogik der Grounded Theory [13] unter Zuhilfenahme der Analysesoftware MaxQDA. Mittels Narrationsanalyse wurden die Fallerzählungen als „Stegreiferzählungen“ sequenzanalytisch und erzählchronologisch ausgewertet.

\section{Limitationen}

Diese explorative Studie bietet aufgrund ihrer qualitativen Methodik einen umfassenden Einblick in die hausärztliche Wahrnehmung des „Ist-Zustandes“

beim stationär-ambulanten Übergang in Deutschland. Es wurde eine zahlenmäßig kleine Anzahl von Hausärzten in Sachsen-Anhalt befragt, daher sind die Ergebnisse nicht verallgemeinerbar auf alle Hausärzte oder andere Fachgruppen.

Eine verlässliche Zuordnung der Fallerzählungen in Gegenwart oder jüngere Vergangenheit ist nicht möglich, Erinnerungseffekte könnten so eine objektiv bestehende Situation überlagern. Trotz Erhebung umfangreichen Interviewmateriales bis zur inhaltlichen „Sättigung“ der Themenkategorien kann nicht von einer vollständigen Abbildung der Problemkonstellationen ausgegangen werden.

\section{Ergebnisse}

Hausärzte nehmen eine zunehmende Versorgungskomplexität bei vulnerablen Patienten seit DRG-Einführung wahr, assoziieren diese aber nicht mit „blutiger Entlassung“ oder „Drehtüreffekt. Stattdessen wird die Entlassungspraxis der Kliniken kritisiert und für Versorgungsbrüche insbesondere bei vulnerablen Patienten verantwortlich gemacht. Vier Themenkategorien wurden in besonders vielen Fallerzählungen berührt (Tabelle 2).

Tabelle 2: Hauptkategorien der Fallerzählungen mit Häufigkeit der Nennungen

\begin{tabular}{|l|c|}
\hline - $\begin{array}{l}\text { (Fehlende) Ankündigung veränderten Versorgungs- } \\
\text { und Unterstützungsbedarfs vulnerabler Patienten }\end{array}$ & 32 von 41 (78 \%) \\
\hline - Unvollständige oder verspätete Entlassungsbriefe & 40 von 41 ( $98 \%)$ \\
\hline $\begin{array}{l}\text { Konfliktträchtige Arzneimittel- und Heil-/Hilfsmittel- } \\
\text { empfehlungen }\end{array}$ & 34 von 41 ( 83\%) \\
\hline $\begin{array}{l}\text { Unzureichende Schulung / Information von Patienten } \\
\text { und Angehörigen }\end{array}$ & 22 von 41 ( $54 \%)$ \\
\hline
\end{tabular}




\subsection{Fehlende Ankündigung veränderten Versorgungs- und Unterstützungsbedarfs}

Die Fallerzählungen zeigten regelhaft, dass Hausärzte erst von einer Krankenhausentlassung erfahren, wenn sich der Patient auf den Weg in die Sprechstunde begibt oder einen um Hausbesuch bittet. "Ich bin wieder da“, so zitiert ein Hausarzt eine Patientin, die sich unmittelbar nach Krankenhausentlassung ungeplant mit komplexem Versorgungsbedarf vorgestellt habe. Kontaktaufnahmen zum Zweck der Entlassungsplanung durch Krankenhäuser, die aus Sicht der Hausärzte in der Verantwortung für die Entlassungsplanung stehen, findet auch in Fallerzählungen zu vulnerablen Patienten zumeist nicht statt: In 32 von 41 Fallschilderungen kritisieren Hausärzte, dass weder eine aus ihrer Sicht erforderliche Vorankündigung des Entlassungszeitpunktes, noch eine Vorabinformation zu veränderten Versorgungs- und Unterstützungsbedarfen erfolgte. Berichtete Gefährdungen der häuslichen Versorgung bezogen sich überwiegend auf unzureichend abgesicherte Verrichtungen des täglichen Lebens (u.a. Grundpflegeleistungen und haushaltsbezogene Unterstützungsbedarfe). Versorgungsbrüche im Zusammenhang mit der Behandlungspflege wurden vor allem in Hinblick auf Wundversorgung berichtet: In 19 von 41 Fallerzählungen traten Probleme der Wundbehandlung bzw. Versorgung mit Verbandsmaterialien (zumeist über das Wochenende hinweg) auf.

Der stationär-ambulante Übergang wurde insbesondere bei Patienten mit einer vorübergehenden oder dauerhaften Unfähigkeit zur Selbstversorgung in eigener Häuslichkeit als problematisch beschrieben. Im Mittelpunkt stand dabei unzureichende Planungszeit für Hausärzte sowie für die Familie des Patienten und/oder ambulante Pflegedienste, um auf einen erhöhten Unterstützungsbedarf zu reagieren.

„Da haben die diesen schwerkranken Mann am Freitagnachmittag nachhause geschickt (!) obne sich zu überlegen (.) ob der überhaupt was zu essen zuhause hat (!). Es war nichts vorbereitet (') ... Es war niemand zuhause der sich um ihn gekümmert hätte. Die Nachbarn haben ihm dann was zu essen gegeben und (.) das sind Dinge (.) die dürfen einfach nicht passieren (!) \& Und das war wieder dasselbe (!) mangelnde Information (.) mangelnde Kooperation (.) (...)auch keine Prüfung des Umfeldes (!) “....,Ich mache der Klinik nicht den Vorwurf, dass sie ihn so entlassen haben (-) das war (.) $z u$ akzeptieren \& Ich hab' / ich mache der Klinik den Vorwurf, dass kein einziger Anruf zu mir kam (') dass ich nicht telefonisch vorbereitet worden bin (') " (Hausärztin / Praxisgemeinschaft Stadt)

\subsection{Unvollständige oder verspätete Entlassungsbriefe}

Konflikte infolge ausbleibender, verspäteter, unvollständiger bzw. fehlerhafter Entlassungsbriefe schilderten 15 von 16 Hausärzten in ihren Fallerzählungen als eine Art anhaltendes „Dauerproblem“.

„(...) und das müssen sich mal überlegen o im Sommer (!) ja (') .. und ich kriege jetzt im Februar den Krankheitsbericht vom Juli (!) Nun brauche ich 'n nicht mehr." (Hausärztin / Einzelpraxis Stadt)

In 11 von 41 Fallerzählungen wird von Entlassungsbriefen berichtet, deren unvollständige Informationen bezogen

\section{Versorgungskontinuität benötigt mehr Planungszeit und bessere Kommunikation.}

auf Diagnosen, Therapien und Dosierempfehlungen Rückfragen erforderlich machten, um Behandlungspfade oder Verordnungen nachvollziehen zu können.

„Das ist der klassische schlechte Fall, weil wir dann einfach keine Hinweise haben (,) keine Medikation haben, keine Therapieempfehlung haben und nichts gar nichts. (...) Oder es sind auch andere Fälle, wo Entlassungsbefunde unkorrekt sind, wo Medikationsangaben unkorrekt sind, wo Milligramm-Angaben fehlen, wo die Dosierung der Medikamente fehlt. das sind also auch relativ häufig vorkommenden Geschichten. das passiert also bei jedem Krankenhaus." (Hausarzt / Einzelpraxis Land)

In 14 von 41 Fallerzählungen gestaltete sich die Suche nach einem auskunftsfähigen Ansprechpartner im Krankenhaus für An- oder Rückfragen schwierig. So berichtete ein Hausarzt von einer Rückfrage zu einer stationär umgestellten Insulintherapie:

„Und dann rufe ich da an wegen der Zuckereinstellung, weil ich das einfach nicht nachvollziehen konnte was se da gemacht haben (...) und dann sagt er: dazu kann ich ibnen keine Auskunft geben' und ich sage: 'na sie haben doch die Krankenakte 'ja doch, aber nicht die Zuckerkarte, die ist bei den Diabetologen'." (Hausarzt / Einzelpraxis Stadt)

\subsection{Konfliktträchtige Arzneiverordnungen und Heil-/ Hilfsmittelempfehlungen}

Hausärzte beschrieben in 22 von 41 Fallerzählungen, dass krankenhausseitige Arzneiverordnungen und/oder Empfehlungen zur Versorgung mit Heil- oder Hilfsmitteln unter den ambulanten Bedingungen (Arbeitsweise, Budgets, Verordnungsrichtlinien) nicht umsetzbar gewesen wären. Dies belaste die Beziehung zwischen Hausarzt und Patient und wirke sich nachteilig auf die Therapietreue aus. Als ein häufig wiederkehrendes schwieriges Problem wird beschrieben, dass die Patienten „mit einer Latte von Medikamenten (...)“ aus dem Krankenhaus kämen „..und ich soll dann jedes Mal 'ne neue Packung und ein neues Medikament aufschreiben." (Allgemeinarzt Einzelpraxis, Großstadt). Auch unterschiedliche Auslegungen von Arzneimittelindikationen bzw. - Zulassungen werden häufig berichtet:

Dann gibt es Patienten (...) die werden in der Klinik mit Medikamenten eingestellt wo / naja auf 'off-label-use' (...) und wir sollen das dann weiter geben, sie hat auf die Verordnung dieses Medikamentes bestanden" (Hausarzt, Einzelpraxis Stadt)

Hausärzte sehen oftmals die Therapietreue (Adherence) ihrer Patienten und damit den Behandlungserfolg gefährdet durch Unklarheiten bzw. Diskontinuitäten in der Arzneiverordnung, wie ein Landarzt berichtet: „...es schafft Unsicherheit für den Patienten, weil ich im Beisein des Patienten anrufen muss, wie viel Medikamente er nehmen muss." (Zitat Landarzt/Einzelpraxis)

Die Befragten beschrieben Rechtfertigungsdruck und Erklärungsnot, wenn sie ihren Patienten Zuzahlungen (Medikamente, Pflegehilfsmittel, Pflegeleistungen) erklären müssen. Berichtet wurde, dass Patienten sich enttäuscht und verunsichert zeigten, wenn sie nach Entlassung vom Hausarzt erfuhren, dass stationäre Verordnungen selbst bezahlt 
werden müssen, Zuzahlungen verursachen oder unter Antrags- bzw. Genehmigungsvorbehalt stehen

„Was aber feststeht ist (') dass zum größten Teil die Krankenhausärzte wenig von den Gepflogenheiten und Arbeitsweisen eines KV-tätigen Arztes kennen (.) ja (') ". (Zitat Hausarzt / Landarztpraxis)" (Hausarzt / Praxisgemeinschaft Stadt)

\subsection{Unzureichende Schulung/ Information von Patienten und Angehörigen}

In insgesamt 34 von 41 Fallerzählungen wird deutlich, dass der stationär-ambulante Übergang im Zusammenhang mit kurzen stationären Liegezeiten durch mangelnde Lernzeiten oder Ressourcennutzung erschwert werden kann. Hausärzte sehen ein erhöhtes Gefährdungspotential, wenn Patienten den Umgang mit ihrer Erkrankung, den damit verbundenen Einschränkungen und therapeutischen Notwendigkeiten während des Krankenhausaufenthaltes nicht nachhaltig erlernen bzw. verstehen konnten.

„Wenn ein insulinpflichtiger Diabeti-

\section{Entlassungspraxis gefährdet die Versorgungskontinuität bei vulnerablen Patienten.}

ker entlassen wird, der aber nicht auf das selber spritzen geschult wurde, der dann sich eventuell noch über's Wochenende mit Insulinspritzen intensiviert versorgen soll (,) gar nicht so richtig weiß wie er das jetzt lernen soll und wie er das jetzt machen soll. (...) das sind dann so Sachen, wo man schon mal überlegt, wenn jemand vierzehn Tage oder drei Wochen im Krankenhaus war, warum solche Sachen da nicht mal angedacht worden sind." (Hausarzt / Einzelpraxis Land)

In 28 von 41 Fallerzählungen wurde geschildert, welchen besonderen Beratungs- und Schulungsbedarf es bedeutet, wenn eine schwere Erkrankung unvorhergesehen einen „vorher gesunden“ Patienten betrifft (z.B. Anus praeter, Katheter, Amputationen). Alle befragten Hausärzte zeigten sich überzeugt, dass eine „ausreichende Lernzeit“ und Patientenschulung vor der Entlassung notwendig sind, um die Grundlagen für Krankheitsbewältigung, Therapietreue und -zufriedenheit zu legen.
/ Wie gesagt, es hat ja eigentlich gar keiner richtig im Krankenhaus mit ihm gesprochen. Es hat ihn keiner aufgeklärt (') es hat eigentlich keiner richtig mit ihm gesprochen. (...) Wie gesagt (.) nie im Krankenhaus gewesen (.) nie große Schmerzangaben gehabt (...)mit 'nem Kolostoma aufgewacht ist (') damit überhaupt nicht ( $k$ ) das überhaupt nicht annehmen wollte (-) (...) \& Ich hab gesagt: „Der Patient ist noch nicht bereit, zuhause alleine klar zu kommen" \& Die Anschlussheilbehandlung dauerte ( $k$ ) 'ne Woche später (.) der schafft das alleine nicht (...) So (.) dann ist er also entlassen worden. (Hausärztin, Einzelpraxis Stadt)

\section{Diskussion}

Hausärzte bringen (empfundene) zunehmende Komplexität der nachstationären Behandlung nicht primär mit sinkender stationäre Verweildauer, G-DRG-Einführung, „blutiger Entlassung“ oder „Drehtüreffekten“ in Verbindung. Kritisiert wird jedoch vielfach die als unzureichend wahrgenommene, Entlassungsvorbereitung bei vulnerablen Patienten mit komplexem Versorgungsbedarf - trotz in Deutschland seit 2012 eingeführter gesetzlicher Verpflichtung zum qualitätsgesicherten Entlassungsmanagement.(\$ 39 GKV-VStG SGB V).

Damit stehen unsere Ergebnisse in deutlichem Kontrast zu quantitativ angelegten Hausarztbefragungen nach G-DRG-Einführung, die über die Wahrnehmung vermehrter „blutiger Entlassungen“ berichteten [8]. Möglicherweise ist dieser Befund auf Lern- und Gewöhnungsprozesse zurückzuführen [5].

\subsection{Ursachen von Versorgungsbrüchen}

Die Analyse der Fallerzählungen unterstreicht eindrucksvoll eine Anzahl aktuell erneut bestätigter Befunde aus Sicht von sowohl Krankenhausärzten, Hausärzten, Patienten und deren Angehörigen zu den Ursachen von Versorgungsbrüchen beim stationär-ambulanten Übergang [14]. Diese entstehen überwiegend durch unzureichende Kooperation bzw. Informationsübermittlung im Rahmen der Entlassungsplanung $[15,16]$. Bei der Überleitung der Patienten von einem Behandlungssektor in den anderen besteht kaum persönlicher Kontakt zwischen den behandelnden Ärzten. Auskunftsfähige Ansprechpartner für Rückfragen des Hausarztes fehlen häufig, obwohl sie ein guter Prädiktor für Zufriedenheit mit den Krankenhausleistungen und ein reibungsloses Entlassungsmanagement sind $[15,16]$.

Als ursächlich hierfür wird, wie auch in unseren Ergebnissen, nicht die fehlende Struktur des Entlassungsmanagements, sondern vielmehr mangelnde Kenntnis und Verständnis seitens der Klinikärzte gesehen [14]. Besonders bei der Anschlussversorgung vulnerabler Patienten konzentriert sich der hausärztliche Unmut gegenüber Kliniken. Wie in internationalen Studien belegt, erwarten die deutschen Befragten ebenfalls nicht „irgendeinen“ Entlassungsbrief, sondern rechtzeitige klare Informationen zum (ggf. veränderten) Versorgungs- und Unterstützungsbedarf mit praxisgerechten Arznei- und Hilfsmittelempfehlungen, die auch anderes Format als 'Briefform' haben können $[17,18]$.

\subsection{Absicherung von Versorgungskontinuität}

Versorgungskontinuität wird erschwert durch „Informationslücken“ gegenüber Hausärzten, Patienten und ihren Angehörigen. Ausschlaggebend für den nach Entlassung von Patienten gesuchten Hausarztkontakt war deren vor Entlassung entwickeltes Verständnis für die Erkrankung und Erfordernisse der Anschlussbehandlung [14, 19]. Hier könnten sich verkürzte Liegezeiten indirekt über verkürzte „Lernzeiten“ zur Krankheitsbewältigung negativ auswirken, wie in den Fallerzählungen benannt. Planbare „Lernzeiten“ über häusliche Verlaufsbegleitung durch Primärversorgungsteams reduzieren dagegen Risiken und Wiederaufnahmeraten [18].

Es reicht nicht aus, die Entlassung vulnerabler Patienten mit komplexem Versorgungsbedarf über die Sozialarbeiter der Klinik planen zu lassen oder Anschlussheil- bzw. Rehabilitationsbehandlungen zu initiieren, sondern ärztliche Expertise und Kooperation sind hierbei [14]. Dafür erscheint es sinnvoll, ärztliche Einschätzungen (aus Klinik und Praxis) zum Versorgungsbedarf vulnerabler Patienten in den wegweisenden „Expertenstandard Entlassungs- 
management in der Pflege“" systematisch mit einzubeziehen [20].

Der Kontakt mit dem Hausarzt in der Entlassungsplanung vulnerabler Patienten nützt jedoch nicht nur Patienten und deren Angehörigen [18, 19]. Zufriedenere Patienten und Hausärzte machen auch Krankenhausärzte zufriedener mit ihrem Beruf [21]. Vor allem aber ist die aktuelle Zufriedenheit mit der Zusammenarbeit der gewichtigste Grund für Hausärzte, in ein bestimmtes Krankenhaus einzuweisen [22].

\subsection{Fazit für die Praxis}

Eine Sicherstellung der Versorgungskontinuität beim stationär-ambulanten Übergang bedarf eines verlässlichen Vorgehens in der Kooperation zwischen Krankenhäusern und Hausärzten mit folgenden Elementen (und möglichen Qualitätsindikatoren):
- Identifikation vulnerabler Patienten und Erfassung / Feststellung von deren nach-stationärer Unterstützungsbedarfe (Alltags- und Krankheitsbewältigung, Hilfs- und Heilmittel, Medikamentenversorgung) rechtzeitig vor Entlassung;

- Übermittlung behandlungswichtiger Informationen im Vorfeld der Entlassung vulnerabler Patienten an den Hausarzt;

- Etablierung einer konstanten Kooperationsstruktur („Hotline“) in der Klinik als koordinierender Instanz, um und auskunftsfähige Ansprechpartner für Hausärzte vorzuhalten;

- Wechselseitige Beteiligung an Qualitätszirkeln und Fortbildungen zum Einweisungs- bzw. Entlassungsmanagement als Basis eines konstruktiven Dialoges z.B. anhand von Fallbesprechungen „kritischer Ereignisse".

\section{Danksagung}

Wir bedanken uns bei allen Interviewpartnern für die ebenso offenen wie engagierten Gespräche und das entgegengebrachte Vertrauen.

\section{Literatur}

\section{Sachverständigenrat zur Begutachtung} der Entwicklung im Gesundheitswesen (Hrsg.). Sondergutachten 2012: Wettbewerb an der Schnittstelle zwischen ambulanter und stationärer Gesundheitsversorgung. Deutscher Bundestag 2012: Drucksache 17/10323

2 Sachverständigenrat zur Begutachtung der Entwicklung im Gesundheitswesen (Hrsg.). Gutachten 2007: Kooperation und Verantwortung - Voraussetzungen einer zielorientierten Gesundheitsversorgung. Deutscher Bundestag 2007: Drucksache 16/6339

3 Rau F, Roeder N, Hensen P (Hrsg.). Auswirkungen der DRG-Einführug in Deutschland. Standortbestimmung und Perspektiven. Stuttgart: Kohlhammer; 2009, S. 17.

4 InEK Institut für das Entgeldsystem im Krankenhaus GmbH (Hrsg.). G-DRG-Begleitforschung gemäß § $17 \mathrm{~b}$ Abs. 8 KHG. Endbericht des dritten Forschungszyklus (2008-2010). Siegburg: InEK Eigenverlag; 2013

\section{Ommen O, Ullrich B, Janßen Ch, Pfaff H.}

Die ambulant-stationäre Schnittstelle in der medizinischen Versorgung. Probleme, Erklärungsmodell und Lösungsansätze. Med Klin 2007; 102:913-7.

\section{Shepperd S, Lannin NA, Clemson LM,} McCluskey A, Cameron ID, Barras SL. Discharge planning from hospital to home (Review) In Cochrane Database Syst Rev. 2013, Issue 1. CD 000313

7 Buhr P, Müller, Braun B, Klinke S, Rosenbrock R. G-DRG und Patienten - Entlassung und Entlassungsmanagement. In: Güntert BJ, Thiele G. (Hrsg) DRG nach Konvergenzphase. Heidelberg: Economia Verlag; 2008.

8 Sens B, Wenzlaff P, Pommer G, von der Hardt H. DRG-induzierte Veränderungen und ihre Auswirkungen auf die Organisation, Professionals, Patienten und Qualität. Zentrum für Qualität und Management im Gesundheitswesen der Ärztekammer Niedersachsen. Hannover: ÄKN Eigenverlag 2009.

9 Berendsen AJ, de Jong GM, Meyboom-de Jong B, Dekker JH, Schuling J. Transition of care: experiences and preferences of patients across the primary/secondary interface - a qualitative study. BMC Health Serv Res. 2009; 9: 62. doi: 10.1186/1472-6963-9-62

10 Tandjung R, Rosemann T, Badertscher N. Gaps in continuity of care at the interface between primary care and specialized care: general practitioners' experiences and expectations. Int J Gen Med. 2011; 4: 773-8

11 Strauss AL. Grundlagen Qualitativer Sozialforschung (2007). (unveränderter Nachdruck der 2. Auflage 1998). München: Wilhelm Fink Verlag, S. 274-93.

12 Meuser M, Nagel U. Das Experteninterview - konzeptionelle Grundlagen und methodische Anlage. In: Pickel S, Pickel G, Lauth $\mathrm{HJ}$, Jahn D ( $\mathrm{Hg})$ Methoden der vergleichenden Politik- und Sozialwissenschaft (2009). Wiesbaden: VS

13 Glaser, B. G., Strauss, A. (1967/2005): Grounded Theory. Strategien qualitativer Sozialforschung. 2. korrigierte Auflage. Bern: Huber 2005

14 Hesselink G, Schoonhoven L, Plas M, Wollersheim H, Vernooij-Dassen M. Quality and safety of hospital discharge: a study on experiences and perceptions of patients, relatives and care providers. Int J Qual Heath Care. 2013; 25(1): 66-74

15 Etesse B et al. How the relationships between general practitioners and intensivists can be improved: The general practitioners' point of view. Critical Care 2010; 14: R 112.

16 Glintborg B, Andersen SE, Dalhoff K. Insufficient communication about medication use at the interface between hospital and primary care. Qual Saf health Care 2007; 16: 34-39.

17 Al-Rashed SA, Wright DJ, Roebuck N, Sunter W, Chrystyn H. The value of inpatient pharmaceutical councelling to elderly patients prior to discharge. Clin Pharmcol 2002; 54: 657 664

18 Rytter L, Jakobsen H N, Ronhld F, Hammer AV, Andreasen AH, Nissen A, Kjellberg J. Comprehensive discharge follow-up in patients homes by GPs and district nurses of elderly patients. Scandinavian Journal of Primary Health Care 2010; 28: 146-153.

19 Yang SC, Zwar N, Vagholkar S, Dennis $\mathrm{S}$, Redmond H. Factors influencing general practice follow up attendances of patients with complex medical problems after hospitalization. Family Practice 2010; 27: 62-68.

20 Möller A. Ein ausgereiftes Entlassungsmanagement entscheidet über den Erfolg der Klinik. In: Kölking H (Hrsg) DGR und Strukturwandel in der Gesundheitswirtschaft. Kohlhammer, S. 374-380.

21 Martinussen PE. Hospital physicians'assess ments of their interaction with GPs: The role of physician and community characteristics. Health Policy 2013; 110(1): 14-21

22 Birk HO, Henriksen LO. Which factors decided general practitioners'choice of hospital on behalf of their patients in an area with free choice of public hospital? A questionnaire study. BMC Health Serv Res. 2012; 12: 126. 